\title{
A CASE FOR ANATOMIC CORRECTION IN ATRIOVENTRICULAR DISCORDANCE? EFFECTS OF SURGERY ON TRICUSPID VALVE FUNCTION
}

Marjan Jahangiri, FRCS

Andrew N. Redington, FRCP

Martin J. Elliott, FRCS

Jaroslav Stark, FRCS

Victor T. Tsang, FRCS

Marc R. de Leval, FRCS
Objective: To assess tricuspid valve function in atrioventricular discordance after palliative procedures (pulmonary artery banding and Blalock-Taussig shunt) and corrective procedures (anatomic and physiologic repair).

Methods: Tricuspid valve dysfunction was assessed by transthoracic echocardiography and graded as no regurgitation (0), mild (1), moderate (2), and severe (3) before and after palliative and corrective procedures performed in 97 patients with atrioventricular discordance between 1988 and 1999. Thirty-two percent had an isolated ventricular septal defect, $43 \%$ had a ventricular septal defect and pulmonary stenosis, and $16 \%$ had pulmonary stenosis. Twenty-six patients underwent pulmonary artery banding and 28 had a Blalock-Taussig shunt. Seventy patients underwent physiologic and 19 underwent anatomic repair. Six patients underwent one-ventricle repair.

Results: After pulmonary artery banding, the tricuspid regurgitation score decreased from $1.7 \pm 0.8$ to $0.9 \pm 0.6(P<.001)$. In patients who underwent a Blalock-Taussig shunt, the tricuspid regurgitation score increased from 0.7 \pm 0.5 preoperatively to $1.4 \pm 0.6$ postoperatively $(P<.001)$. After physiologic repair, there was no significant change in the tricuspid regurgitation score; however, 7 patients required additional repair or replacement. The regurgitation score was significantly reduced from $1.5 \pm 0.8$ to $0.4 \pm 0.5$ $(P<.001)$ after anatomic repair. The operative mortality in patients who underwent physiologic repair was $7 \%$ as compared with $0 \%$ in the anatomic repair group $(P=.59)$. The median follow-up was 3.2 years.

Conclusions: Right ventricular volume loading (shunt) worsens tricuspid regurgitation, whereas volume reduction (banding) or left-to-right septal shift (anatomic repair) has beneficial effects. We have not observed a significant change in the tricuspid regurgitation score after physiologic repair. Anatomic repair can be performed in selected patients with atrioventricular discordance and provides superior functional results. (J Thorac Cardiovasc Surg 2001;121:1040-5)
A trioventricular discordance is commonly associated with lesions including ventricular septal defect (VSD), pulmonary stenosis, and tricuspid valve abnormalities. Surgery in these patients has classically resulted in a physiologic repair, whereby the systemic circulation is supported by the morphologically right

From the Cardiothoracic Unit, Great Ormond Street Hospital for Children NHS Trust, London, United Kingdom.

Read at the Eightieth Annual Meeting of The American Association for Thoracic Surgery, Toronto, Ontario, Canada, April 30-May 3, 2000.

Copyright (c) 2001 by The American Association for Thoracic Surgery

0022-5223/2001 $\$ 35.00+0 \quad \mathbf{1 2 / 6 / 1 1 3 1 7 4}$

doi:10.1067/mtc.2001.113174 ventricle. Even in the presence of a structurally normal tricuspid valve, right ventricular dysfunction and tricuspid regurgitation (TR) can develop after physiologic repair. ${ }^{1}$ Furthermore, the reported morbidity and mortality after operations in patients with an abnormal tricuspid valve has been high. ${ }^{2,3}$ These factors have led to the introduction of the double switch procedure or anatomic repair, in which normal atrioventricular and ventriculoarterial connections are restored., ${ }^{4,5}$ Isolated reports of improved tricuspid valve function after pulmonary artery banding $(\mathrm{PAB})$ and anatomic repair have been published. ${ }^{6}$

We set out to investigate the effects of palliative procedures (PAB and Blalock-Taussig shunt) and corrective procedures (anatomic repair and physiologic 
Table I. Associated anomalies in 97 patients with atrioventricular discordance

\begin{tabular}{lc}
\hline Anomaly & $n$ \\
\hline Isolated VSD & $31(32 \%)$ \\
VSD and PS & $42(43 \%)$ \\
PS & $16(17 \%)$ \\
\hline
\end{tabular}

$V S D$, Ventricular septal defect; $P S$, pulmonary stenosis.

repair) on tricuspid valve function in patients with atrioventricular discordance.

\section{Patients and methods}

A retrospective analysis of patients with atrioventricular discordance who underwent surgery between January 1988 and August 1999 was carried out. A total of 97 patients were identified. The cohort includes patients who had a palliative procedure before 1988 and were admitted for further surgery between 1988 and 1999.

Anatomic variations. Situs solitus was present in 85 (89\%) patients, situs inversus in $11(11 \%)$ patients, and 1 patient had left atrial isomerism. Eighty-three $(86 \%)$ had levocardia, $11(11 \%)$ had dextrocardia, and 3 (3\%) patients had mesocardia. Nine patients had situs solitus and dextrocardia, 1 of whom underwent anatomic repair.

The ventriculoarterial connection was discordant in 88 (91\%), and 9 (95) patients had double-outlet right ventricle. Two patients had coarctation of the aorta and 14 patients had an atrial septal defect. Eighteen (19\%) patients had rhythm abnormalities before any intervention. Associated anomalies are shown in Table I.

\section{Surgical procedures}

$P A B$. PAB was performed in 26 patients. Eleven patients subsequently underwent anatomic repair at a median interval of 3.2 years (range 7 months-6.3 years). Only 1 of these patients had undergone a preliminary PAB with the intention to prepare for a double switch operation. Thirteen patients underwent physiologic repair at a median interval of 3.7 years (range 3 months-10.4 years). Two patients have not undergone any further surgery and are awaiting a double switch operation.

Blalock-Taussig shunt. A Blalock-Taussig shunt was performed in 28 patients, 3 of whom underwent anatomic repair at a median interval of 3.7 years (range 1.8-3.7 years) and 25 of whom underwent physiologic repair at a median interval of 5.8 years (range 1 month- 10.3 years).

The anatomic repair only became available in the latter part of the series. The patients who were operated on in the earlier part were only considered for physiologic repair.

Physiologic repair. Seventy patients underwent physiologic repair at a median age of 11.2 years (range 6 months-35 years). Physiologic repair comprised all procedures that maintained the morphologically right ventricle supporting the systemic circulation. This included VSD closure in 25 patients, direct relief of pulmonary valvular or subvalvular
Table II. Surgical procedures performed in patients who underwent physiologic repair

\begin{tabular}{lc}
\hline Procedure & $n$ \\
\hline VSD closure & 25 \\
LV-PA conduit & 17 \\
VSD closure + LV-PA conduit & 12 \\
Pulmonary valvotomy/relief of subvalvular stenosis & 6 \\
VSD closure + pulmonary valvotomy & 3 \\
TV repair/replacement & 7 \\
\hline
\end{tabular}

$V S D$, Ventricular septal defect; $L V-P A$, left ventricle-pulmonary artery; $T V$, tricuspid valve.

stenosis in 6 patients, VSD closure and pulmonary valvotomy in 3 patients, use of a left ventricle-pulmonary artery conduit in 17 patients, and VSD closure and the use of a conduit in 12 patients. Seven patients underwent tricuspid valve repair or replacement (Table II). Four of the 12 patients who underwent VSD closure and use of a conduit and 8 of the 17 patients who had undergone a left ventricle-pulmonary artery conduit have subsequently been reoperated on for conduit obstruction at a median age of 6.9 years.

Anatomic repair. Nineteen patients underwent anatomic repair at a median interval of 9.1 years (range 6.5 months-19 years). Fifteen patients had an associated VSD and underwent an atrial and arterial switch (double switch), a Mustard switch operation in 7 and a Senning switch operation in 8 . Four patients had VSD and pulmonary stenosis: Two underwent a Mustard-Rastelli procedure and 2 a Senning-Rastelli operation.

One-ventricle repair. Six patients underwent a "Fontan repair." Five had a Fontan operation because of ventricular hypoplasia: small right ventricle in 4 patients and small left ventricle in 1 . Another patient with a horizontal septum, a VSD that was remote from both great vessels, and abnormal attachments of tricuspid valve chordae was thought to be unable to undergo repair.

Other procedures. Five patients underwent insertion of a pacemaker for congenital heart block, and 1 patient had heart transplantation.

\section{Surgical technique}

VSD closure. In the majority of cases, the VSD is closed through the right atrium, the sutures being placed on the morphologically right side of the septum as described previously. ${ }^{7}$ If the VSD is subaortic, it may be closed through the aorta. Rarely, the VSD can be closed through the left atrium in cases of situs solitus with dextrocardia or situs inversus with levocardia.

Left ventricle-pulmonary artery conduit. Care is taken in making the incision in the left ventricle to avoid injury to the anterior papillary muscle of the mitral valve. Before the incision is made, the left ventricle is inspected through the mitral valve to avoid damaging the papillary muscle and to identify the site of the ventriculotomy.

In physiologic repair, for placement of the conduit, the left ventricle-pulmonary artery conduit is placed to the right side of the aorta in situs solitus and to the left side of the aorta in 


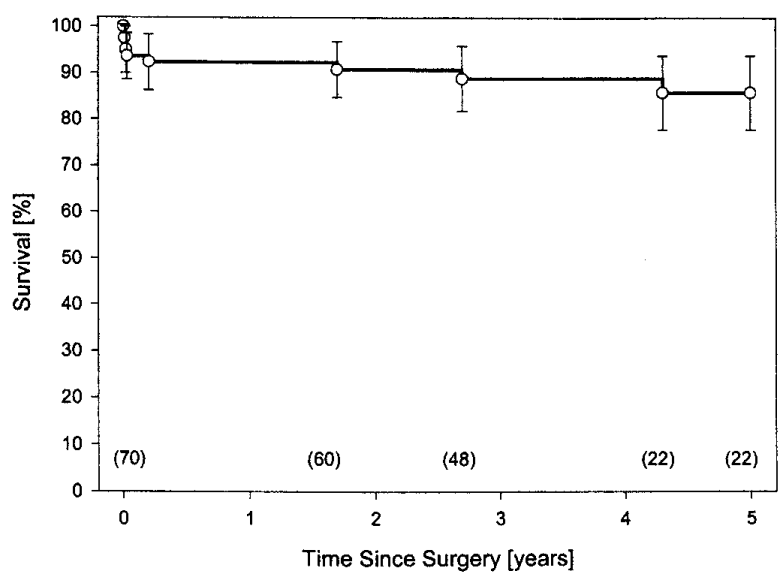

Fig 1. Kaplan-Meier survival curve for patients who underwent physiologic repair.

situs inversus. For anatomic repair, in patients with situs solitus, the conduit is placed away from the sternum and to the left of the aorta. The conduit is anastomosed end to side to the main pulmonary artery.

Tricuspid valve anatomy and function. The anatomy of the tricuspid valve was assessed by cross-sectional transthoracic echocardiography. The tricuspid valve was denoted Ebstein-like if there was displacement of the septal leaflet and dysplastic if the leaflets were thickened.

Tricuspid valve dysfunction was assessed by spectral and color Doppler transthoracic echocardiography. Dysfunction was subjectively graded as follows, both before and after surgery: no TR $=0$, mild $=1$ (a narrow regurgitant jet reaching no further than mid atrium and not associated with left atrial or right ventricular dilatation), moderate $=2$ (a regurgitant jet reaching the atrial wall or associated with left atrial or right ventricular dilatation), and severe $=3$ (a broad jet associated with obvious failure of tricuspid valve apposition and left atrial and right ventricular dilatation). This dysfunction was expressed as the TR score. The mean TR score for each group was calculated.

Statistical analysis. The outcome variables were survival and TR. The paired Student $t$ test was used to compare preoperative and postoperative TR score. Actuarial estimates for survival time and freedom from tricuspid valve dysfunction were determined by the Kaplan-Meier product-limit method with $95 \%$ Greenwood confidence intervals. The SAS software package version 6.12 (SAS Institute, Inc, Cary, NC) was used for all statistical analysis.

\section{Results}

Survival. Six patients died in the hospital after the operation. All deaths occurred after physiologic repair. There were no early deaths after anatomic repair or in patients who underwent one-ventricle repair. This difference was not significant. The actuarial survival for all patients at 1, 3, and 5 years was $94 \%, 90 \%$, and

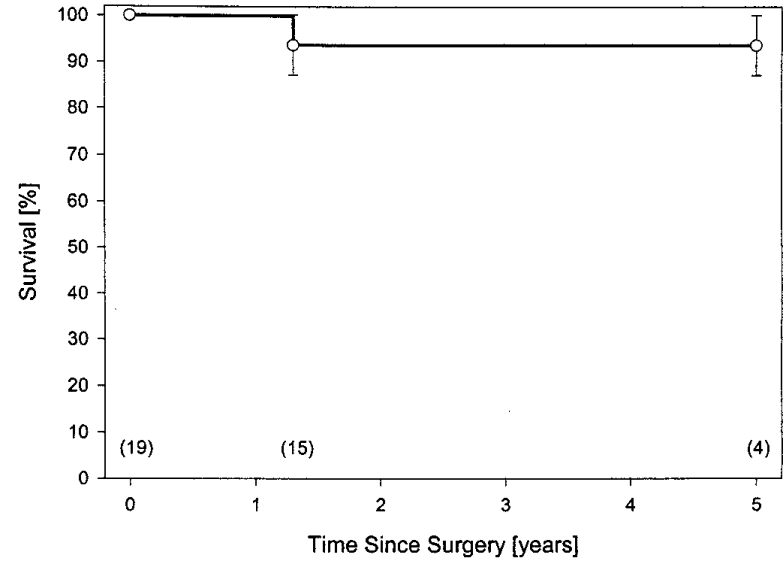

Fig 2. Kaplan-Meier survival curve for patients who underwent anatomic repair.

$87 \%$, respectively (Figs 1 and 2). There were 3 late deaths in the physiologic repair group and 1 late death in the anatomic repair group. The actuarial survival for patients who underwent physiologic repair at 1,3 , and 5 years was $93 \%, 90 \%$, and $86 \%$, respectively (Fig 1). The actuarial survival for patients who underwent anatomic repair at 3 and 5 years was $93 \%$ (Fig 2).

Tricuspid valve anatomy and function. Eight patients had Ebstein anomaly of the tricuspid valve and 4 had dysplasia. The changes in TR score in each subgroup after surgery are shown in Table III. The mean TR score before and after surgery is shown in Table IV.

$P A B$. TR score was available in 25 of the 26 patients who underwent PAB. The mean TR score was $1.7 \pm 0.8$ before banding and $0.9 \pm 0.6$ after banding $(P<.001)$.

Two patients underwent $\mathrm{PAB}$ in the last 2 years of the study to reduce severe TR, and in both the TR improved significantly. They have not yet undergone any further operation. Fig 3 shows a transesophageal echocardiogram demonstrating dynamic changes in TR in 1 of these patients. It illustrates the shift of the interventricular septum with virtual abolition of TR.

Blalock-Taussig shunt. The TR score was available in 22 of the 28 patients who underwent a Blalock-Taussig shunt. The TR score increased from $0.7 \pm 0.5$ before the shunt to $1.4 \pm 0.6$ after the operation $(P<.001)$.

Physiologic repair. The TR score was available in all patients having physiologic repair. Overall, the TR score decreased from $1.5 \pm 0.9$ before to $1.3 \pm 0.8$ after the operation. This decrease did not reach statistical significance. Seven patients in the physiologic repair group had severe TR necessitating repair or replacement of the tricuspid valve. The TR score in this group decreased from $2.9 \pm 0.4$ to $1.4 \pm 1.1(P=.02)$. 


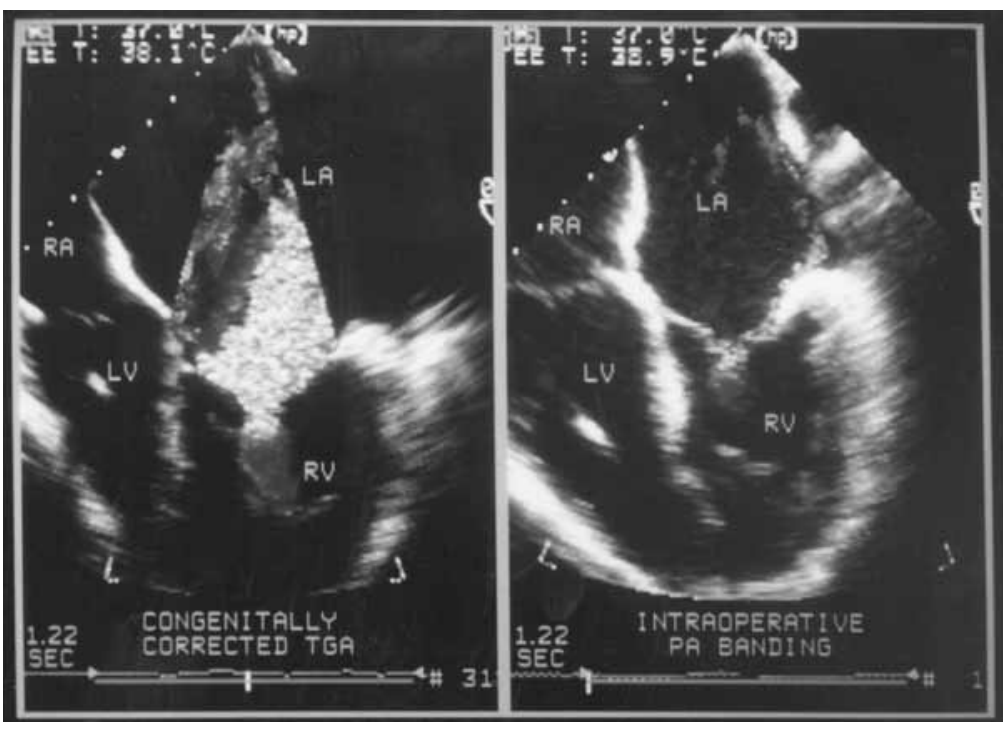

Fig 3. Transesophageal echocardiogram demonstrating dynamic changes in TR. The septum has shifted and the TR has disappeared. $T G A$, Transposition of the great arteries; $P A$, pulmonary artery; $L A$, left atrium; $L V$, left ventricle; $R A$, right atrium; $R V$, right ventricle.

Table III. The changes in TR score after surgery in each subgroup

\begin{tabular}{lcccc}
\hline Operation & $n$ & Unchanged & Increased & Decreased \\
\hline PAB & 25 & 14 & 0 & 11 \\
BT shunt & 22 & 9 & 12 & 1 \\
PR & 76 & 57 & 7 & 12 \\
AR & 19 & 8 & 1 & 10 \\
\hline
\end{tabular}

$\overline{P A B}$, Pulmonary artery banding; $B T$, Blalock-Taussig shunt; $P R$, physiologic repair; $A R$, anatomic repair.

Anatomic repair. TR score was also available in all patients having anatomic repair. The mean preoperative TR score in this group was $1.5 \pm 0.8$. This decreased significantly to $0.4 \pm 0.5$ after repair $(P=.001)$. The TR score after anatomic repair was significantly less than that after physiologic repair $(P<.0001)$.

Tricuspid valve repair/replacement. Four patients underwent tricuspid valve repair and 3 patients underwent tricuspid valve replacement. All 7 patients were in the physiologic repair group. Three of the patients had Ebstein-like anomaly of the tricuspid valve. One infant who had closure of VSD and an attempted repair at 2.5 months continued to have severe TR. In view of his age, replacement was not considered. He underwent removal of the VSD patch, atrial septectomy, creation of tricuspid valve atresia with a patch, and PAB. This patient is still awaiting further surgery. One of the patients who underwent mechanical valve replacement had undergone repair 4 years previously.
Table IV. Mean tricuspid valve score before and after surgery

\begin{tabular}{|c|c|c|c|c|}
\hline Operation & $n$ & $\begin{array}{c}\text { Preop } \\
\text { TR score }\end{array}$ & $\begin{array}{l}\text { Postop } \\
\text { TR score }\end{array}$ & $\mathrm{P}$ value \\
\hline РAB & 25 & $1.7 \pm 0.8$ & $0.9 \pm 0.6$ & $<.001$ \\
\hline BT shunt & 22 & $0.7 \pm 0.5$ & $1.4 \pm 0.6$ & $<.001$ \\
\hline PR & 76 & $1.5 \pm 0.9$ & $1.3 \pm 0.8$ & .27 \\
\hline $\mathrm{AR}$ & 19 & $1.5 \pm 0.8$ & $0.4 \pm 0.5$ & .001 \\
\hline TV rep/repl & 7 & $2.9 \pm 0.4$ & $1.4 \pm 1.1$ & .02 \\
\hline
\end{tabular}

$P A B$, Pulmonary artery banding; $B T$, Blalock-Taussig shunt; $P R$, physiologic repair; $A R$, anatomic repair; $T R$, tricuspid regurgitation, $T V$ rep/repl, tricuspid valve repair/replacement.

Requirement for pacemaker. New heart block developed in 9 patients who underwent physiologic repair and in 1 patient who had anatomic repair. All required insertion of a pacemaker.

\section{Discussion}

The results of physiologic repair have been disappointing, with significant morbidity occurring both early and late after the operation. ${ }^{8}$ Progressive TR, right ventricular dysfunction, atrioventricular block, and conduit-related problems have all been reported. These problems led to the introduction of anatomic repair, restoring the right ventricle and the tricuspid valve to their usual subpulmonary positions. Our data suggest that this strategy is beneficial to tricuspid valve function, which appears to be variable depending on 
volume load of the systemic right ventricle and the septal position.

Lundstrom and coworkers ${ }^{9}$ examined the severity of TR in patients with atrioventricular discordance and in relation to the volume load on the right ventricle. They showed a low incidence of TR in patients with naturally occurring pulmonary stenosis.

In our study, we found a significant worsening of TR after Blalock-Taussig shunting and improvement in those undergoing PAB. This is probably due to the effects of, respectively, increased and decreased right ventricular preload with concomitant changes in left atrial and right ventricular volume rather than septal shift. As we discuss below, movement of the interventricular septum may be important in some, but the majority of our patients had an associated VSD, with the interventricular septum in a neutral position, making this mechanism an unlikely candidate for the change observed after palliative procedures.

In the current study, PAB was most frequently performed when a VSD was present to reduce pulmonary blood flow. However, 3 patients with intact ventricular septum underwent PAB to prepare for a double switch operation. One has subsequently undergone anatomic repair. The concept of using PAB to prepare the left ventricle to support the systemic circulation was first introduced for transposition of the great arteries. ${ }^{10}$ Van Son and colleagues ${ }^{11}$ have reported on 5 patients with previous Mustard or Senning operations for simple transposition of the great arteries in whom systemic ventricular failure and TR subsequently developed. $\mathrm{PAB}$, performed as an interim step toward an arterial switch operation, led to a marked reduction of TR, which decreased still further after anatomic repair. ${ }^{11}$

Our data confirm a similar significant improvement in tricuspid valve function after anatomic repair in patients with congenitally corrected transposition of the great arteries. Similar observations were made by Imai and Momma $^{5}$ in patients with atrioventricular discordance undergoing anatomic correction; five of the 50 patients died, but TR disappeared in 20 of the 22 patients with significant preoperative TR, which in turn led to a significant reduction in right ventricular enddiastolic volume.

We have concentrated mainly on early outcome and function of the tricuspid valve after anatomic repair. The long-term implications of intra-atrial repair are widely known in those treated for simple transposition of the great arteries. In addition, the attrition of extracardiac conduits placed underneath the sternum in patients who have undergone the atrial-Rastelli procedure and the risk of heart block are issues when deciding on surgical strategy. On balance, both anatomic and physiologic repair have potential long-term problems.
Some of these issues are avoided by physiologic repair, and we must point out that, overall, tricuspid valve function did not change after physiologic repair. However, 4 patients required tricuspid valve repair and 3 required replacement, 1 of whom had undergone a previous repair. In our study, physiologic repair did not create TR when this was not present preoperatively. However, we believe that if TR is present at the outset, it has to be dealt with. Furthermore, the higher morbidity and mortality associated with physiologic repair, as well as the long-term uncertainties regarding the ability of the right ventricle to function as a systemic ventricle, add to the attractiveness of anatomic repair, particularly in the presence of significant TR or pre-existing right ventricular dysfunction. Our current policy is to perform anatomic repair in patients with atrioventricular and ventriculoarterial discordance, especially in those with TR or reduced systemic ventricular (morphologically right ventricle) function.

In atrioventricular discordance, TR increases after a Blalock-Taussig shunt and decreases after palliation by PAB. Overall, TR is unaffected by physiologic repair, although approximately $10 \%$ of the patients required tricuspid valve repair/replacement. We believe that anatomic repair can be performed with low mortality and significant reduction in TR.

We thank Mr Christopher Lincoln for one third of the patients from his Royal Brompton Hospital practice and also Dr Francoise and Dr Sluysman from Belgium for some of the patients undergoing anatomic correction.

Received for publication May 9, 2000; revisions requested July 13, 2000; revisions received Nov 3, 2000; accepted for publication Nov 28, 2000.

Address for reprints: Marc R. de Leval, FRCS, Cardiothoracic Unit, Great Ormond Street Hospital for Children NHS Trust, Great Ormond St, London WC1N 3JH, United Kingdom (E-mail: marc.deleval@goshtr.nthames.nhs.uk).

\section{REFERENCES}

1. Sano T, Risenfeld T, Karl TR, Wilkinson JL. Intermediate-term outcome after intracardiac repair of associated cardiac defects in patients with atrioventricular and ventriculoarterial discordance. Circulation 1995;92(Suppl):II-272-8.

2. Horvath P, Szufladowicz M, de Leval MR, Elliott MJ, Stark J. Tricuspid valve abnormalities in patients with atrioventricular discordance: surgical implications. Ann Thorac Surg 1994;57: 941-5.

3. Williams WG, Suri R, Shindo G, Freedom RM, Morch JE, Trusler GA. Repair of major intracardiac anomalies associated with atrioventricular discordance. Ann Thorac Surg 1981;31:527-32.

4. Ilbawi MN, DeLeon SY, Backer CL, Duffy CE, Muster AJ, Zales VR, et al. An alternative approach to the surgical management of physiologically corrected transposition with ventricular septal 
defect and pulmonary stenosis or atresia. J Thorac Cardiovasc Surg 1990;100:410-5.

5. Imai Y, Momma K. Double switch operation in 50 patients with congenitally corrected transposition of the great arteries. Proceedings of the Second World Congress of Paediatric Cardiology and Cardiac Surgery in Hawaii. 1997. p. 20-22.

6. Acar P, Sidi D, Bonnet D, Aggoun Y, Bonhoeffer P, Kachaner J. Maintaining tricuspid valve competence in double discordance: a challenge for the paediatric cardiologist. Heart 1998;80:479-83.

7. de Leval MR, Bastos P, Stark J, Taylor JF, Macartney FJ, Anderson RH. Surgical technique to reduce the risks of heart block following closure of ventricular septal defect in atrioventricular discordance. J Thorac Cardiovasc Surg 1979;78:515-26.

8. Yeh T, Connelly MS, Coles JG, Webb GD, McLaughlin PR, Freedom RM, et al. Atrioventricular discordance: results of repair in 127 patients. J Thorac Cardiovasc Surg 1999;117:1190-203.

9. Lundstrom U, Bull C, Wyse R, Somerville J. The natural and unnatural history of congenitally corrected transposition. Am J Cardiol 1990;65:1222-9.

10. Yacoub MH, Radley-Smith R, Maclaurin R. Two-stage operation for anatomical correction of transposition of the great arteries with intact interventricular septum. Lancet 1977;1:1275-8.

11. van Son JA, Reddy M, Silverman NH, Hanley FL. Regression of tricuspid regurgitation after two-stage arterial switch operation for failing systemic ventricle after atrial inversion operation. J Thorac Cardiovasc Surg 1996;111:342-7.

\section{Discussion}

Dr Tom R. Karl (Philadelphia, Pa). Can you provide any information about right ventricular function and tricuspid valve anatomy in the patients in whom TR developed after repair? I do not think the problem of right ventricular dysfunction can be considered separately from TR in discordant transposition of the great arteries.

I noticed that the risk of heart block was very low in the patients undergoing anatomic repair. Could you comment on the technique of VSD closure? Was this done through a great artery or through the mitral valve, using the de Leval technique, or by some other technique?

On the basis of your clinical experience, would you now consider anatomic repair to be the best option for all suitable patients with discordant transposition of the great arteries, even those who have good tricuspid valve anatomy and function and good biventricular function? Should the operation be offered only to suboptimal candidates for classic repair or to all patients?

Finally, are there some patients for whom PAB might be considered a suitable long-term solution?

Dr Jahangiri. Dr Karl, thank you for your comments and your questions.

Regarding right ventricular dysfunction, 7 patients underwent tricuspid valve repair or replacement. Of the 4 patients who underwent tricuspid valve repair, 2 have moderate TR with moderate right ventricular dysfunction. I agree with you that right ventricular dysfunction is a very important issue. It is hard to know which comes first-the TR or the right ventricle dysfunction. Of the 3 patients who underwent tricuspid valve replacement, 1 who had had a previous repair now has gross right ventricular dysfunction and is currently listed for transplantation. Despite the fact that the postoperative TR score in these patients looks satisfactory, they have moderate to severe right ventricular dysfunction and on the whole are not doing well.

Regarding your second question about heart block and VSD closure, if the VSD is subaortic, we tend to approach it through the aorta. Otherwise, the approach is through the atrium. If a Rastelli procedure is required in the presence of pulmonary stenosis, when a ventriculotomy is performed, then we close the VSD through the ventricle. The conduction tissue has been defined by Professor Marc de Leval and Robert Anderson in their articles from the 1980s, and we try to avoid this during VSD closure.

Regarding the choice of the anatomic or physiologic repair, when TR is present, the patient is a suitable candidate for anatomic repair, and if the surgeon has the expertise to perform this procedure, anatomic repair is a good option. However, if TR is not present and the morphologic features are difficult-isomerism, dextrocardia, difficult venous connections-then physiologic repair remains a suitable option.

Dr Christo I. Tchervenkov (Montreal, Quebec, Canada). I would like to raise two anatomic or technical issues. Patients with situs solitus and dextrocardia present a surgical challenge in terms of performance of the atrial switch if an anatomic repair is considered. This is due to the smaller and rather inaccessible right atrium because of the dextrocardia. In such patients, we have performed a modified Senning operation by using an in situ "pericardial well" technique along with the anatomic intracardiac repair. How did the presence of dextrocardia in the face of situs solitus influence your decision of whether to perform an anatomic or a physiologic repair?

The second issue pertains to the placement of the right ventricle-pulmonary artery conduit. Reoperation for conduit change can be tricky because the conduit often is compressed directly behind the sternum. At the original operation, is the conduit placed to the left or to the right of the aorta?

Dr Jahangiri. Regarding your question about dextrocardia and the situs solitus, we use an anatomic repair. I suppose we have been lucky. None of our 19 patients has had dextrocardia.

In physiologic repair, the left ventricle-pulmonary artery conduit is placed to the right side of the aorta in situs solitus and to the left of the aorta in situs inversus. In anatomic repair and situs solitus, this is placed to the left of the aorta. However, during redo operations, I found the conduit to be right in the middle behind the sternum and quite difficult to deal with. In these patients we elect to go on bypass before sternotomy.

Dr John E. Mayer (Boston, Mass). I have a question about the patients who had the Fontan operations. What happened to tricuspid valve function in the patients who had a preceding Blalock-Taussig shunt, presumed volume load of the ventricles, and then underwent the Fontan procedure? Once the volume load was relieved, what happened to the tricuspid valve or regurgitation?

Dr Jahangiri. I regret, I do not have the data available to answer your question. 